\title{
LA ESCUELA NACIONAL SUPERIOR DE ENFERMERAS DE COLOMBIA. RELACIONES CON ORGANISMOS INTERAMERICANOS
}

\author{
Ana Luisa Velandia Mora
}

Licenciada en Enfermería por la Universidad Nacional de Colombia

Profesora Emérita, Universidad Nacional de Colombia

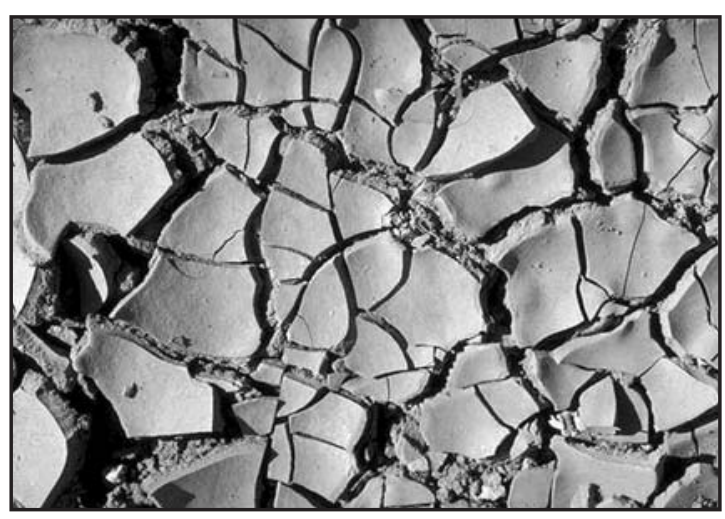

THE NATIONAL HIGH SCHOOL OF NURSES OF COLOMBIA. RELATIONS WITH INTERAMERICAN ORGANISMS

\section{SUMMARY}

$\mathrm{T}$ This writing is a contribution to the project: "Formation of personnel of infirmary in the National University of Colombia", that carries out the Group of Investigation in Nursing History in the same University. This work has as objective present history the National Nursing High School of the National University of Colombia - ENSE, from its creation in 1943 to its transformation in Faculty of Infirmary, in 1958. The National Nursing High School is an emblematic institution in the Colombian infirmary and is part of a representative time of the direction of the health services towards an approach hygienist, promoted in all the continent by inter-American organisms.

This phenomenon happens in a time in a context characterized by great legal development in nursing, as much by prescribed laws of the profession, like by prescribed decrees of the nursing schools, and especially by the legal prerogatives that granted to the National Nursing High School.
On the other hand, this school can be considered as one of the pillars of the implantation in Colombia of the american influence that arrived from the United States and Canada, countries that had received "the nightinguiliana" influence, of English nurses, in second half of Century XIX.

Key words: nursing, education, Colombia, National University of Colombia.

A ESCOLA NACIONAL SUPERIOR DE ENFERMEIRAS DE COLÔMBIA. RELAÇÕES COM ORGANISMOS INTERAMERICANOS

\section{RESUMO}

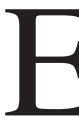
Iste escrito é um contribua ao projecto: "Formação de pessoal de enfermagem na Universidade Nacional de Colômbia", que leva a cabo o Grupo de Investigação em História da Enfermagem da mesma Universidade. O artigo tem como objectivo dar a conhecer a história da Escola Nacional Superior de Enfermagem da Universidade Nacional de Colômbia - ENSE, desde sua criação em 1943 até sua transformação em Faculdade de Enfermagem, em 1958. A Escola Nacional Superior de Enfermagem é uma instituição emblemática na enfermaria colombiana e faz parte de uma época representativa da orientação dos serviços de saúde para um enfoque higienista, promovido em todo o continente por organismos interamericanos. Corresponde ademais a uma época de grande desenvolvimento legal da enfermagem, dado tanto por leis regulamentares da profissão, como por decretos regulamentares das escolas de enfermagem, e especialmente pelas prerrogativas legais que se lhe concederam à Escola Nacional Superior de Enfermeiras. Por outra parte, 
esta escola pode-se considerar como um dos pilares da implantação em Colômbia da influência americana que chegou desde Estados Unidos e Canadá, países que tinham recebido a influência "nightinguiliana", de enfermeiras inglesas, na segunda metade do Século XIX.

Palavras Chave: enfermagem, educação, Colômbia, Universidade Nacional de Colômbia.

\section{RESUMEN}

$\mathrm{E}$ ste escrito es un aporte al proyecto: "Formación de personal de enfermería en la Universidad Nacional de Colombia", que lleva a cabo el Grupo de Investigación en Historia de la Enfermería de la misma Universidad. El artículo tiene como objetivo dar a conocer la historia de la Escuela Nacional Superior de Enfermeras de la Universidad Nacional de Colombia - ENSE, desde su creación en 1943 hasta su transformación en Facultad de Enfermería, en 1958. La Escuela Nacional Superior de Enfermeras es una institución emblemática en la enfermería colombiana y hace parte de una época representativa de la orientación de los servicios de salud hacia un enfoque higienista, promovido en todo el continente por organismos interamericanos. Corresponde además a una época de gran desarrollo legal de la enfermería, dado tanto por leyes reglamentarias de la profesión, como por decretos reglamentarios de las escuelas de enfermería, y especialmente por las prerrogativas legales que se le concedieron a la Escuela Nacional Superior de Enfermeras. Por otra parte, esta escuela se puede considerar como uno de los pilares de la implantación en Colombia de la influencia americana que llegó desde Estados Unidos y Canadá, países que habían recibido la influencia "nightinguiliana", de enfermeras inglesas, en la segunda mitad del Siglo XIX.

Palabras claves: enfermería, educación, Colombia, Universidad Nacional de Colombia.

\section{Organización de los Servicios de Salud}

Con las ayudas prestadas por la Fundación Rockefeller, desde 1914 en Costa Rica, Nicaragua, Guatemala, Panamá y El Salvador; los referentes norteamericanos tuvieron gran influencia en el establecimiento de modelos sanitarios adoptados por gobiernos nacionales, entre ellos el de Colombia.

Quevedo y colaboradores (1990), denominan la etapa de la salud en Colombia 1886 - 1947, como el "Modelo Higienista". En septiembre de 1942, el gobierno colombiano pide participación en el programa interamericano de cooperación y, para tal efecto, Nelson Rockefeller visita al país, igualmente el Dr. Howard B. Schooknoff, quien venía destinado para ser el Jefe del SCISP- SCISP.

La profundización de la influencia del modelo higienista en salud se hace especialmente significativa en la formación del recurso humano. (Eslava, 2004). El SCISP, en su programa de labores en 1943 acordó algunas campañas sanitarias, entre otras la lucha contra el paludismo, y otras actividades como la fundación del Instituto Nacional de Nutrición y la construcción del edificio para la escuela de enfermeras (Bejarano, 1948).

Para Restrepo y Villa (1980), el SCISP “montó en el Ministerio de Salud Pública una estructura vertical, dirigida y administrada por técnicos norteamericanos con la colaboración de colombianos que se iban formando para este fin... el cual rompió la orientación de atención integral de la fase anterior, por la separación rotunda entre los aspectos preventivos a cargo del subsector oficial y los curativos bajo la responsabilidad de la caridad".

El hecho más importante en salud en esta época, lo constituye la Ley $6^{\mathrm{a}}$. de 1945 que se ha considerado el primer Estatuto de Seguros Sociales en Colombia y por medio de la cual se había creado la Caja Nacional de Previsión para los trabajadores al servicio del Estado. En 1946 y mediante la Ley 90 se crea el Instituto Colombiano de Seguros Sociales (ICSS), adscrito al Ministerio de Trabajo, Higiene y Previsión Social (Abel, 1996). Según Hernández (2002), paralelamente a la creación del ICSS se da el debate en torno a la necesidad de un ministerio para la salud. En 1945 Jorge Bejarano, entonces Ministro de Trabajo, Higiene y Previsión Social, presenta al Congreso un proyecto en tal sentido, y se crea el Ministerio de Higiene (Ley 27 de 1946).

Los centros de salud, las enfermerías y los orfelinatos se desarrollaron de manera desigual en las diferentes regiones del país y fueron víctimas fáci- 
les de cierres cuando los ajustes fiscales así lo exigieron. Sin embargo, las campañas patrocinadas por la Fundación Rockefeller se imponían sin consultar a los propietarios y trabajadores rurales que eran sus principales beneficiarios (Abel, 1996).

\section{Desarrollo de la Educación}

Según Helg (1989), hasta 1958 la política educativa nacional fue orientada a frenar el éxodo rural de los jóvenes y a asegurar una educación separada para las mujeres. La controversia en torno a las orientaciones de la enseñanza media se hizo candente en 1937 al establecerse el examen oficial obligatorio para el ingreso a las universidades. Conservadores y liberales se sintieron furiosos por la influencia que Carmen Rodrigo, psicóloga y refugiada comunista de España, ejercía sobre el escaso número de cupos de la Universidad Nacional. Según Abel (1996), un grupo de profesores hizo un llamado para que la Universidad Javeriana abriera su Facultad de Medicina, argumentando que era indispensable que los estudiantes asistieran a clases obligatorias de moral y ética católicas. Algo similar sucedió en enfermería. Las diferencias en su ejercicio por parte de las órdenes religiosas que implicaba el cumplimiento de ciertas obligaciones establecidas por su comunidad religiosa, y por parte de los profesionales laicos, que aspiraban a horarios de trabajo y salarios de acuerdo a su formación, fue un tema de controversia entre los administradores hospitalarios.

\section{Presencia de Organismos Interamericanos}

En 1942 fueron enviadas al país por la Oficina Sanitaria Panamericana dos enfermeras, Hellen Howitt, canadiense, y Johanna Schwarte, norteamericana; para que asesoraran en materia de enfermería al Ministerio de Trabajo, Higiene y Previsión Social, en sus campañas higiénicas asistenciales. (Pedraza, 1942).

En el Memorándum de antecedentes sobre la fundación de la Escuela Nacional Superior de Enfermeras (Memorándum de Antecedentes, 1975), se expone:

"Nuestras enfermeras se educan en ambiente hospitalario para atender enfermos, pero están desconectadas de la parte social y desde luego carecen de una apreciación sobre el alcance de nuestras campañas sanitarias y de ahí el que con estos ele- mentos sea imposible hacer una transformación educativa tanto en el ramo de la sanidad como en el de la nutrición y protección de la infancia, que vamos a iniciar."

"Los doctores Pedraza y Aconcha resolvieron de común acuerdo interesar a las dos enfermeras extranjeras para crear una Escuela Nacional de Enfermeras con el fin de formar un personal que pudiera dirigir otras escuelas y colaborar eficazmente en la Campaña Materna - Infantil" (Wiesner, 1976).

Al definir la financiación para su funcionamiento, en el Memorándum se plantea:

"La Oficina Sanitaria Panamericana costearía las expertas (enfermeras extranjeras), y además ofrecería las becas necesarias para complementar estudios en Estados Unidos. La Fundación Rockefeller costearía el personal científico nacional; y la alimentación, servicios y dotación serían atendidos por el Servicio de Salubridad (Servicio Cooperativo Interamericano de Salud Pública) en cooperación con el gobierno colombiano" (Memorándum de Antecedentes, 1975). La financiación para la construcción de la Escuela se haría con un aporte adicional por parte del Grupo General Brigadier Dunham. (Del Ejército de los Estados Unidos).

\section{La Escuela Nacional Superior de enfermeras}

El Presidente López Pumarejo expide 4 de Marzo de 1943 el Decreto 466, por el cual se crea la Escuela Nacional Superior de Enfermeras, dependiente del Ministerio de trabajo, Higiene y Previsión Social y supervigilada por la Universidad Nacional. Se regiría por una Junta Directiva de 5 miembros y solamente recibiría alumnas con diploma de enseñanza secundaria (Bachiller o Normalista), convirtiéndose en la primera escuela que exigió diploma de enseñanza secundaria completa en Colombia y probablemente en toda América Latina.

Para dirigirla fue nombrada la señorita Hellen Howitt, quien según Álvarez (2005), era egresada de la Universidad de Alberta (Canadá). Según información encontrada en Internet (Foundationnysnurses, 2005), Helen Howitt egresó de Belleuve Hospital School of Nursing en 1923. Las primeras cinco instructoras fueron seleccionadas entre las primeras graduandas de la Escuela 
Nacional de Enfermeras. Además de su salario, contaban además con residencia (Documento Bodas de Oro, 1975), (Wiesner, 1975).

Una de las Instructoras, Rosa Sáenz, era egresada de la Escuela de Enfermeras del Hospital Santo Tomás de Panamá, tal como ella nos lo contó (Sáenz, 1989):

"Helen Howitt, antes de venir a Colombia, fue Directora de la Escuela de Panamá en el tiempo que yo estaba de estudiante; cuando a Helen Howitt la nombraron para venir a organizar la escuela de aquí, ella se acordó de mí."

El Acta de la Sesión Inaugural del Comité Ejecutivo de la Escuela Nacional Superior de Enfermeras, del 12 de mayo de 1943, nos aporta los siguientes datos:

"Luego de la lectura por parte del doctor Luis E. Aconcha de la Resolución No. 431 del 6 de los corrientes, el Dr. Schooknoff pregunta: por qué se pone 'bachiller o por lo menos normalista?'... El doctor Aconcha hace la aclaración de que existen dos bachilleratos, uno superior y otro inferior y es unánimemente aprobada la modificación de poner 'bachillerato superior o normalista $\operatorname{regular}^{11} \mathrm{La}$ señorita Howitt interviene para preguntar cuánto cuesta la beca que se va a asignar a las alumnas y el Dr. Orozco (Secretario General del Ministerio) responde que es de $\$ 40.00$. (En ese momento, la tasa real de cambio del dólar era de \$1.47)... El Dr. Aconcha propone que podría aprovecharse la gira de los doctores Schooknoff y Orozco, para visitar las escuelas de enfermeras existentes en el país y estudiarles sus condiciones actuales, para dictar posteriormente normas que regulen sus estudios y expedición de diplomas; pues dijo tener conoci-

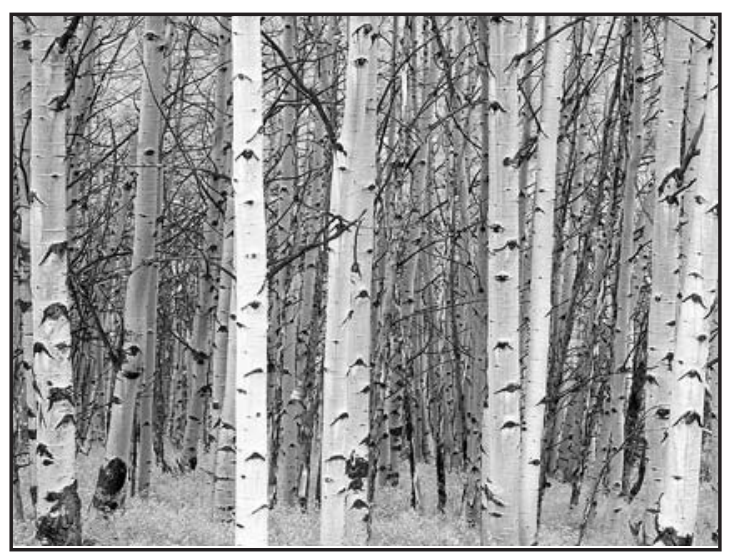

miento de la anarquía que existía en sus pensums (sic) y en la manera de expedir los diplomas" (Acta Comité Ejecutivo ENSE, 1943). Y efectivamente, más adelante se dictaban algunas disposiciones reglamentarias sobre Escuelas de Enfermeras y otras. (Decreto 3151 de 1946).

Conforme a la concepción de la época, en que las mujeres debían estudiar internas (incluso quienes tenían su familia en la misma ciudad); se construyó un edificio en los predios de la ciudad universitaria (OPS, 2002). Las estudiantes tenían derecho a los servicios de bienestar estudiantil que se habían implementado en la Universidad Nacional, como resultado de la reforma de la educación superior (Romero, 1989), (Ferro, 1989). En abril de 1948 se efectúa el traslado a dicha edificación, en la cual funcionó hasta 1968 (Velandia, 2006).

Dos prestantes médicos nos legaron su visión acerca de la historia de la enfermería en Colombia. Según Héctor Pedraza (1954), en desarrollo del proyecto $\mathrm{N}^{\circ} 52$ con apoyo del SCISP, del Ministerio de Trabajo, Higiene y Previsión Social y del Gobierno Nacional se levanta para la Escuela en predios de la Ciudad Universitaria en lote donado para ello por la Universidad Nacional, un edificio construido y dotado con todas los requerimientos técnico-arquitectónicos y de equipamiento para la enseñanza de la enfermería y para vivienda de estudiantes y profesoras.

Según Bejarano (1948) "puede asegurarse, sin exageración alguna, que sus edificios son de los mejores dedicados a este fin en América Latina. Tanto las construcciones dedicadas a vivienda de alumnas como de profesoras, los equipos y casinos, lavandería, laboratorios y demás materiales de enseñanza, no dejan nada que desear; han venido de los Estados Unidos y el costo total de la obra con sus elementos excede de un millón de pesos colombianos; pero en esta forma quedará ya asegurada la existencia definitiva de la enseñanza de la enfermería en Colombia”.

Según Álvarez (2001), estudiante y más tarde profesora de la Escuela: "Helen Howitt, gestionó y aceleró, con la ayuda de la Fundación Rockefeller, la construcción de la bellísima sede para nuestra escuela en los predios de la Ciudad Universitaria, en la que fueron considerados todos los detalles: capilla, cafetería con instalaciones modernas, 
buses, campos de deporte, salones de clase, lavandería, sala de recepciones y visitas con hermoso piano de cola, laboratorios químico y de dietética y sala de demostraciones y artes de enfermería; las habitaciones de alumnas como de profesoras, elegantemente dotadas, apartamento privado para la Directora, en fin, hasta el más mínimo detalle, fue considerado para nuestro confort."

Howitt elaboró un escrito que fue enviado el 5 de julio de 1950 a Miss Mary Alberti, de la Oficina Sanitaria Panamericana. La Revista Avances en Enfermería de la Facultad de Enfermería de la Universidad Nacional de Colombia, publicó una traducción del mismo en diciembre de 2005 (Howitt, 2005), que había sido publicado en noviembre de 1951 en The American Journal of Nursing.

Es bueno conocer la visión que la misma Helen Howitt (2005), tuvo de esta Escuela: "Solo tres de las escuelas existentes, son aprobadas por el Gobierno Nacional - la Escuela de Enfermería de la Cruz Roja Nacional y la Escuela Nacional Superior de Enfermería. (Habla de tres escuelas, pero no menciona sino dos. Por esa época, las otras dos escuelas existentes eran la de la Universidad de Cartagena y la de la Universidad Javeriana) ${ }^{2}$ De las últimas mencionadas, la Escuela Nacional Superior de Enfermería es la más nueva de las tres. Fue abierta en Bogotá en marzo de 1944 con un curso de 43 estudiantes. Cada estudiante presentó un diploma que indicaba que había terminado satisfactoriamente la escuela secundaria, la cual es comparable con el "High School" de los Estados Unidos".

"Esta escuela de tres años fue establecida por el Gobierno Nacional con la cooperación de la Oficina Sanitaria Panamericana, el Instituto de Asuntos Internacionales y la Fundación Rockefeller. En 1950, la escuela con 6 años de fundada, tiene 76 graduadas, 11 de las cuales han realizado estudios de postgrado en los Estados Unidos y han regresado a la Escuela en calidad de profesoras. En 1950, hay 96 estudiantes en la Escuela".

"El plan de estudios está basado en el Currículo Guía de 1937, establecido por la Liga Nacional de Enfermería (de los Estados Unidos). Los laboratorios y salones de clase de la escuela son atractivos y bien equipados".

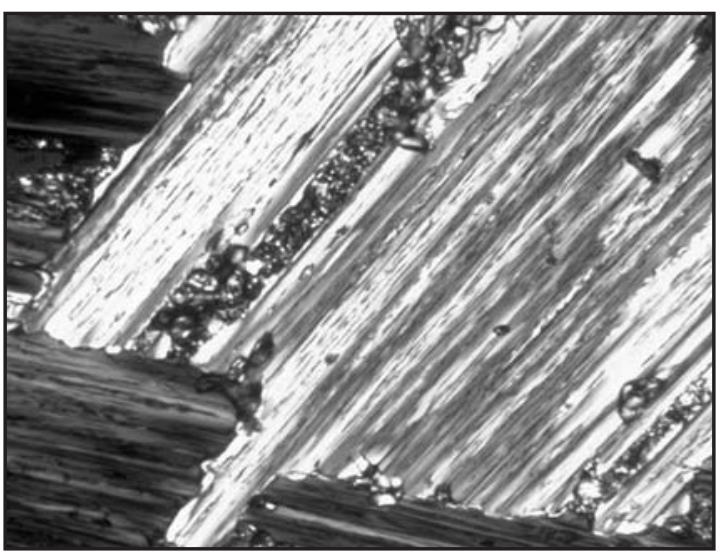

"Las experiencias clínicas son proporcionadas por el Hospital San José, un hospital general de 500 camas. Aquí hay salas de 30 camas para pacientes de medicina, cirugía, ginecología, y divisiones de obstetricia que han sido especialmente equipadas con fines docentes. Hay también cinco salas de operaciones, donde las estudiantes aprenden técnicas quirúrgicas y un recientemente organizado departamento de consulta externa que ofrece muchas oportunidades de aprendizaje".

"Para las experiencias en enfermería pediátrica, las estudiantes van al Hospital de la Misericordia, una institución infantil de 450 camas, donde 40 camas (Pabellón Barranquilla), están reservadas para proveer experiencia clínica a las estudiantes. En cada sala donde las estudiantes practican hay enfermeras jefes, pagadas por la escuela, quienes las enseñan y supervisan".

"Durante el tercer año, todos los estudiantes tienen 3 meses de práctica en un centro de salud pública, donde hay enseñanza y supervisión por enfermeras graduadas. Las estudiantes de enfermería usualmente tienen cinco a seis horas de trabajo en sala y dos o tres horas diarias de clase en aula. Tienen un día libre a la semana. Las actividades curriculares incluyen grupos de canto, deportes, baile y aprendizaje de algún instrumento musical. Una completa y bella nueva residencia para los estudiantes fue abierta en 1948".

"Las estudiantes llegan a la Escuela Nacional Superior de Enfermería (sic) casi de todos los Departamentos de Colombia. Usualmente entran con una beca que es provista bien sea por el Departamento o el Gobierno Nacional. En reciprocidad por la beca, la estudiante acepta retornar a su 
localidad cuando se gradúe y laborar allí por lo menos dos años".

"Enfermería, como profesión, es todavía muy joven en Colombia, pero se está desarrollando marcadamente rápido. Porque hay una gran necesidad de enfermeras preparadas, un número de cursos cortos han sido iniciados para entrenar ayudantes y parteras. Ellas trabajan en los centros de salud, hospitales y clínicas y están contribuyendo a mejorar los programas de salud de Colombia".

Murphey, por su parte, en el artículo que escribió sobre la historia de la enfermería en Colombia (1956), se refiere en los siguientes términos a ésta Escuela:

"El 13 de Marzo de 1944 la Escuela Nacional superior de Enfermeras abrió sus puertas al primer grupo de 43 estudiantes, cuya credencial era el Diploma de Bachiller o Normalista Superior... El Instituto de Asuntos Interamericanos, el SCISP y la Fundación Rockefeller, cooperaron con el gobierno nacional en la organización de esta escuela. La organización, programas y cursos fueron planeados igual que en la más moderna y progresista escuela en los Estados Unidos, y las instructoras y supervisoras han sido enviadas a los Estados Unidos para cursos de post-grado en enfermería... Actualmente el SCISP está edificando en el Hospital San José, un nuevo pabellón que será para que las estudiantes hagan sus prácticas de psiquiatría... La más moderna y bella residencia fue construida por el SCISP en los prados de la Ciudad Universitaria, donde se inspiran en una atmósfera sana y de devoción al estudio."

Las estudiantes en la práctica clínica daban atención directa a los pacientes en turnos que cubrían las 24 horas, inclusive días festivos y dominicales, en los hospitales de San José y la Misericordia, y a partir de 1953, en la Clínica particular de Marly. Se contaba además con el Centro de Salud $N^{\circ} 8$ para la experiencia en salud pública. (Wiesner, 1976), (Velandia, 2009).

El 21 de Febrero de 1947 se graduó un grupo de 23 alumnas, quienes recibieron el título de "Enfermera General" en el Aula Máxima de la Facultad de Medicina de la Universidad Nacional de Colombia, el cual se les refrendó después de 2 años de servicios. En 1946 se había promulgado la Ley 87, que reglamentó los títulos de enfermería y ya se habían aceptado oficialmente las auxiliares de enfermería (diversificadas) y las ayudantes o asistentes.

En el Decreto Presidencial No. 3708 de 1950, y en el Acuerdo 79 de 1951 del Consejo Directivo de la Universidad Nacional, se especifica que la Escuela Superior de Higiene y la Escuela Nacional Superior de Enfermeras, como dependencia de aquella, pasan a formar parte de la Universidad Nacional (Velandia, 2008).

La Escuela Nacional Superior de Enfermeras tuvo dos planes de estudio. Por el Decreto 466 de 1943, se establece un Plan de Estudios de tres años, con Tesis y Examen de Grado. Cada uno de los tres años, distribuido en un primer y un segundo periodo. Si comparamos este primer plan de estudios con el establecido por el Acuerdo No. 5 de 1937 para la Escuela Nacional de Enfermeras (Gómez, 2006), podemos analizar varios aspectos: uno, primordial, tiene que ver con la orientación "sanitaria" o de corte higienista, que incluye una asignatura novedosa: "servicio rural de ayuda a la familia"; otro, tiene que ver con la presencia de asignaturas relacionadas con fisioterapia y nutrición, ya que por esa época las enfermeras también desempeñaban esos roles porque todavía no existían las carreras de fisioterapia y nutrición, de manera independiente de la enfermería. El Acuerdo 14 de 1954 del Consejo Académico de la Universidad Nacional, estableció un nuevo Plan de Estudios para la escuela, con tres años de duración, de dos semestres académicos cada uno. Las asignaturas están organizadas por Grupos, cada uno de los cuales incluye varias asignaturas. Es todavía más orientado a la Salud Pública y revelador de lo que sería más adelante el desarrollo de la enfermería de salud pública en Colombia. Por esta época, en la Escuela de Salud Pública de la Universidad Nacional, bajo los auspicios de la Oficina Sanitaria Panamericana y la UNICEF, se abrió un curso postbásico en Obstetricia con una duración de 8 meses, bajo la dirección de Helen Hentsoel con ayuda de la enfermera colombiana, Rubby Palacio. (Arango, 1966), (Buriticá, 1989).

"La influencia de las fundaciones Kellogg, Rockefeller y Ford en las concepciones y orientaciones prácticas del ejercicio de la profesión que fueron reproducidas de las enfermeras norteameri- 


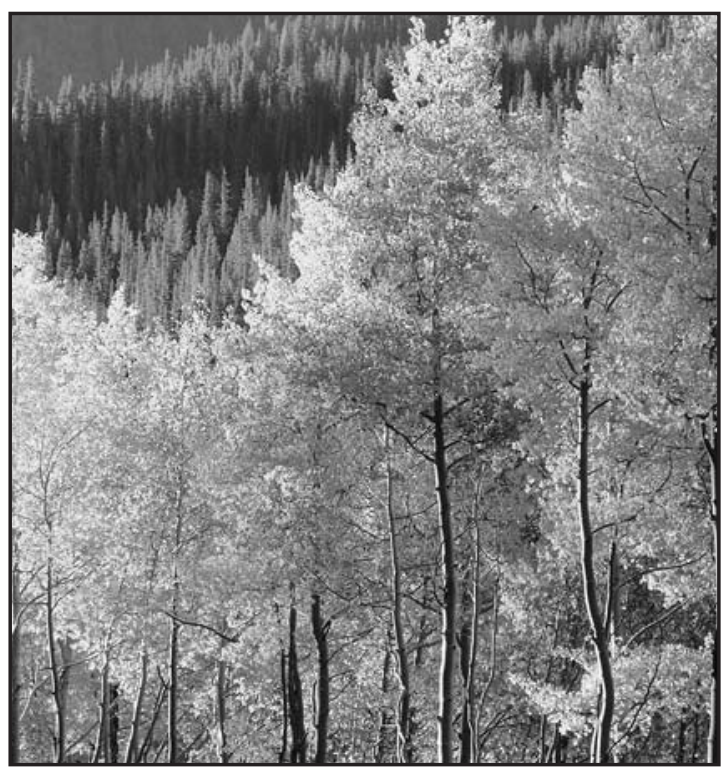

canas, se hizo patente en estos programas", dice Romero Ballén (1992).

Howitt fue directora hasta el año de 1951, la sucedió Katerine Kain, quien no la ocupó mucho tiempo; luego vino la enfermera norteamericana Hellen Murphey, quien la desempeñó asesorada por la enfermera colombiana María Teresa Murillo Pombo (Pedraza, 1954). Helen Murphey estuvo hasta 1955 y durante 1956 y 1957, Murillo Pombo; con quien regresa la dirección de la escuela a enfermeras colombianas. (Wiesner, 1976).

Inés Durana (1992), en su presentación al ser admitida como miembro de la Academia Nacional de Medicina), decía: “Otra mención que en justicia debe hacerse, es la del SCISP(SCISP), brazo internacional del Ministerio de Trabajo, Higiene y Previsión Social” y continúa: "El SCISP coordinó esfuerzos con la Oficina Sanitaria Panamericana y la Fundación Rockefeller, para crear en 1942 la Escuela Nacional Superior de Enfermeras, que concederá el título de Enfermera General, donde se fusionan las dos grandes tendencias de la época, por un lado la formación hospitalaria (que venía teniendo la Escuela de Enfermeras de la Universidad Nacional desde 1937) y por el otro la salubrista..." (Que se venía promoviendo desde los servicios de salud del estado). (Las complementaciones son nuestras). La influencia que en la medicina preventiva tuvo el SCISP es mostrada por diversos autores. (Cuellar, 2006).
Con base en las prerrogativas sobre los demás instituciones universitarias del país, concedidas por el Decreto 260 de 1936, todos los programas de enfermería que nacieron posteriormente, se nutrieron de aquel (Bejarano, 1948). Es así, como se reorganiza la Escuela de Enfermeras y Parteras de Cartagena, la cual en 1949 pasa a ser una dependencia académica de la Universidad de Cartagena. De la misma forma, en 1950 la Universidad Nacional se encargaría de la dirección y organización técnicas y refrendaría los títulos de la Escuela de Enfermería Hospitalaria de la Universidad del Valle.

\section{A manera de conclusiones}

La Escuela Nacional Superior de Enfermeras, representa entonces, la tercera etapa de la enfermería en la Universidad Nacional de Colombia y nació como respuesta a una necesidad del gobierno de preparar enfermeras con orientación comunitaria para sus programas, especialmente en el área materna - infantil; y por las repercusiones que tuvo tanto en la formación como en la práctica de la enfermería en el país, se constituye en un hito de la enfermería colombiana.

Por su parte, Helen Howitt representa un estilo de enfermera en la cual se reflejan las características de su época: la tradición de tipo religioso, la relación de la enfermería con la condición femenina y la herencia militar. (Velandia, 1995, b). Una personalidad que pintan muy bien quienes la conocieron: rígida, pero a la vez muy humana. $\mathrm{Su}$ influencia en la enfermería colombiana fue decisiva. En la Escuela Nacional Superior de Enfermeras, que ella contribuyó a crear y de la cual fue su primera Directora, dejó su impronta de enfermera salubrista en cuanto al enfoque conceptual de su plan de estudios, y de enfermera con formación anglosajona en su organización curricular.

Este modelo continuó hasta una nueva reforma de la educación de enfermería a partir de 1959 , coincidiendo con la creación de la Facultad de Enfermería de la Universidad Nacional de Colombia que fue la primera en conceder el título de Licenciada en Enfermería en un país de habla hispana. "Se realizó entonces un intercambio auspiciado por el gobierno de los Estados Unidos con la Universidad Católica de América en la ciudad de 
Washington, D. C. y se obtuvieron suficientes becas para estudios de postgrado en el exterior", relata Durana (1989).

Como puede deducirse, la década del 50 es muy significativa para la enfermería colombiana, especialmente por los cambios que se dan dentro de la Universidad Nacional.

La influencia de las organizaciones interamericanas en Enfermería, no fue sólo en Colombia. Hellen Howitt fue Directora de la Escuela de Enfermería del Hospital Santo Tomás de la Zona del Canal de Panamá entre 1933 y 1938 (Roy, 2005); luego fundadora y primera Directora de la Escuela Nacional Superior de Enfermeras de Colombia entre los años 1943 y 1951; posteriormente de la Escuela Nacional de Enfermeras de Bolivia entre 1953 y 1959, cuando fue invitada a cumplir un cargo similar en Venezuela. A todos estos países, llegó primero como consultora del respectivo Ministerio de Salud, a través de convenios con el SCISP (Velandia, 2006, b).

Finalmente, la influencia extranjera en la enfermería colombiana no se circunscribe a la influencia americana; a mi manera de ver, los distintos momentos, se podrían clasificar así:

- 1900 a 1924 Influencia francesa (Cartagena, Bogotá)

- 1924 a 1943 Influencia americana, especialmente en la administración de servicios

(Cartagena), y en el enfoque hospitalario (Bogotá)

- 1943 a 1958 Influencia de organizaciones sanitaristas de procedencia americana (SCISP, OPS, Fundación Rockefeller, AID, Fundación W. K. Kellogg)

- 1960 a 1980 Profundización de la Influencia que ya se venía recibiendo del sistema universitario americano, con la llegada de enfermeras colombianas con postgrados en los Estados Unidos (Velandia, 2005).

Sin embargo, desde mediados del Siglo XX se empieza a observar una influencia inter-regional entre países de habla hispana del continente americano. Tal vez el país que empezó a influir más fue Chile, donde se ofrecieron cursos de postgrado, mejor decir postbásicos, a los cuales asistieron enfermeras de diversos países. Más adelante, en la década del 60 , Colombia ofreció el programa de
Licenciatura en Enfermería y tuvo estudiantes procedentes desde México hasta Argentina y en la década del 70 ofreció programas de Maestría, inicialmente en Educación y en Administración en Enfermería, a los cuales también llegaron enfermeras de diferentes países (Velandia, 1995); por su parte México ofreció programas de especialización, especialmente en Pediatría que fueron muy acreditados en toda la región; en la década del 80 , Brasil empezó a ofrecer programas de doctorado, a los cuales empezaron a asistir enfermeras extranjeras, especialmente en la década del 90. Desde la década del 90 y especialmente a comienzos de este siglo, se siente que España está tratando de retomar influencia en la enfermería latinoamericana, especialmente a través del apoyo a sus organizaciones gremiales y de programas de doctorado.

\section{BIBLIOGRAFÍA}

- Abel, Christopher (1996) Ensayos de Historia de la Salud en Colombia, 1920 -1990. Bogotá: Instituto de Estudios Políticos y Relaciones Internacionales de la Universidad Nacional de Colombia - CEREC.

- Acta de la Sesión Inaugural del Comité Ejecutivo de la Escuela Nacional Superior de Enfermeras. Bogotá, Mayo 12 de 1943. Archivo preparado por el Comité Académico para conmemorar las Bodas de Oro de la Carrera de Enfermería de la Universidad Nacional de Colombia, 1925 - 1975. Bogotá, 1975.

- Acuerdo No. 14 de 1954 (Marzo 8). Acta No. 9 del Consejo Académico de la Universidad Nacional de Colombia, "por el cual se determina el plan de estudios para la Escuela Nacional de Enfermeras, dependiente de la Escuela Superior de Higiene". Aprobado por el Consejo Directivo en sesión de Marzo 15 de 1954.

- Acuerdo 79 de 1951 (Abril 25), Acta No. 23 del Consejo Directivo de la Universidad Nacional, en el cual se especifica que la Escuela Superior de Higiene y la Escuela Nacional Superior de Enfermeras pasan a formar parte de la Universidad Nacional.

- Álvarez de Espitia, Lelly. Intervención en la Facultad de Enfermería de la Universidad Nacional de Colombia, con motivo de la Inauguración de la Galería de Retratos de las Directoras y Decanas de las Escuelas y luego Facultad de Enfermería. Bogotá, Mayo 18 de 2001.

- Álvarez de Espitia, Lelly. Entrevista concedida a Ana Luisa Velandia Mora, el 13 de Mayo de 2005.

- Arango T., Bertha (1996) "Reseña histórica de la enfermería de salud pública en Colombia”. Rev. ANEC, Bogotá, Año 1, No. 2, Mayo - Agosto, Págs. 15 - 17.

- Bejarano, Jorge (1948) "Historia y desarrollo de la enfermería en Colombia". Revista de la Facultad de Medicina de la Universidad Nacional de Colombia. Bogotá, Vol. XVII, No. 1, Julio Páginas 1075 - 1079.

- Buriticá, Josefina. Entrevista concedida a Ana Luisa Velandia, en Bogotá, en septiembre de 1989. 
- Cuellar-Montoya, Zoilo. "Académica Inés Durana Samper". Se encuentra en: www.encolombia.com/medicina/academiamedicina//academ26467-inesdurana.htm Consultado: $14 / 03 / 2006$

- Decreto No. 466 de 1943 (Marzo 4), por el cual se crea la Escuela Nacional Superior de Enfermeras. Diario Oficial, Año LXXXVIII, No. 25202. Viernes 12 de Marzo de 1943, Pág. 811.

- Decreto 3151 de 1946 (Octubre 31), por el cual se dictan algunas disposiciones reglamentarias sobre Escuelas de Enfermeras y otras. Diario Oficial, Año LXXXII, No. 26275, página 481 .

- Durana Samper, Inés. Entrevista concedida a Ana Luisa Velandia, en Bogotá, en septiembre de 1989.

- Durana Samper, Inés (1992) "Reflexiones sobre Enfermería". Revista Medicina. Bogotá: Academia Nacional de Medicina. No. 31, Diciembre, pp. 27 - 34.

- Eslava Castañeda, Juan Carlos (2004) Buscando el reconocimiento profesional. La salud pública en Colombia en la primera mitad del siglo XX. Bogotá: Universidad Nacional de Colombia, Págs. 62 - 67.

- Ferro, Pepa. Entrevista concedida a Ana Luisa Velandia, en Bogotá, en septiembre de 1989.

- Garzón Alarcón, Nelly. "Análisis de la experiencia docente como profesora de la Facultad de Enfermería desde 1958 hasta 1976. Bogotá, Universidad Nacional de Colombia, 1977.

- Gómez Serrano, Consuelo (2006) "La Escuela Nacional de Enfermeras de la Universidad Nacional de Colombia: 1937 1944". Revista Avances en Enfermería (Bogotá), Vol. XXIV, No. 2, Julio - Diciembre, Págs. 99 - 109.

- Helg, Aline (1989) "La educación en Colombia. 1946 - 1957". EN: Nueva Historia de Colombia, Bogotá, Planeta Colombiana Editorial, Tomo IV: 111 - 134.

- Hernández Álvarez, Mario (2002) "Muchas lógicas de la política de salud en el proceso de incorporación: 1930 - 1938” EN: La Salud Fragmentada. Bogotá: Universidad Nacional de Colombia: Instituto de Salud Pública - Facultad de Medicina, Págs. 83 - 165.

- Howitt, Helen. (1951) Nursing School in Bogota, S. A., The American Journal of Nursing, Vol. 51, No. 11 (Nov.), pp. 644645, doi:10.2307/3468465. This article consists of 2 page(s).

- Howitt, Helen (2005) "Escuela de Enfermería en Bogotá, S. A." Traducción y comentarios de Ana Luisa Velandia Mora. Revista Avances en Enfermería. (Bogotá). ISSN: 0121 - 4500. Vol. XXIII, No. 2, Julio - Diciembre, pp. 107 - 108.

- Memorándum de Antecedentes sobre la fundación de la Escuela Nacional Superior de Enfermeras. Bogotá, s. f. Archivo preparado por el Comité Académico para conmemorar las Bodas de Oro de la Carrera de Enfermería de la Universidad Nacional de Colombia, 1925 - 1975. Bogotá, 1975.

- Murphey, Helen (1956) "Historia de la Enfermería en Colombia." Revista de la Asociación Mexicana de Enfermeras. Año III, No. 5, Septiembre - Diciembre. Págs. 10 - 12.

- Organización Panamericana de la Salud. La Organización Panamericana de la Salud y el Estado Colombiano: cien años de historia 1902 - 2002. Bogotá, OPS, 2002. Pág. 99.

- Pedraza M., Héctor. (1942) "Informe para el VIII Congreso Panamericano del Niño, sobre organización y actividades de la protección materna e infantil en Colombia". Revista de Higiene, 23 (6-7), Junio - Julio: 10 - 35.
- Pedraza, Héctor (1954) La Enfermería en Colombia. Reseña histórica sobre su desarrollo. Legislación. Bogotá: Editorial Minerva. Tercera Época 1930 - 1950. Págs. 34 - 57.

- Poveda Ramos, Gabriel (1989) "Cien años de ciencia colombiana." En: Nueva Historia de Colombia. Bogotá: Planeta Colombiana Edit.,. Tomo IV. Pp. 159 - 188.

- Quevedo, Emilio y colaboradores. La Salud en Colombia. Análisis Sociohistórico. Primera Parte. Bogotá: Ministerio de Salud - Departamento Nacional de Planeación, 1990. Disponible

en: http://www.saludcolombia.com/actual/salud54/informe.htm. Consultado: 13/04/2009.

- Quevedo, Emilio y colaboradores. Café, Gusanos, Mosquitos y Petróleo. Bogotá. Universidad Nacional de Colombia. Facultad de Medicina. Instituto de Salud Pública, 2004. Págs. $183-185$.

- República de Colombia. Ley 36 de 1942 (Diciembre 2), por la cual se crea el Servicio Cooperativo Interamericano de Salud Pública.

- República de Colombia. Ley 6 de 1945 (febrero 19), por la cual se dictan algunas disposiciones sobre convenciones de trabajo, asociaciones profesionales, conflictos colectivos y jurisdicción especial del trabajo.

- República de Colombia. Ley 27 de 1946 (Diciembre 2), por el cual se crea el Ministerio de Higiene.

- República de Colombia. Ley 87 de 1946 (Diciembre 26), por la cual se reglamenta la profesión de enfermería y se dictan otras disposiciones.

- República de Colombia. Ley 90 de 1946 (Diciembre 26), por la cual se crea el seguro social obligatorio y se crea el Instituto Colombiano de Seguros Sociales.

- Restrepo Ch., Guillermo y Villa V., Agustín. Desarrollo de la Salud Pública Colombiana. Medellín: Universidad de Antioquia, 1980. Pág. 85.

- Romero Ballén, María Nubia. "Modelo pedagógico en enfermería: Una proyección del papel histórico de la mujer". Trabajo de Investigación de Año Sabático: Tunja: Universidad Pedagógica y Tecnológica de Colombia, 1992. Documento de circulación interna.

- Romero Pabón, Concepción. Entrevista concedida a Ana Luisa Velandia, en Bogotá, en septiembre de 1989.

- Roy, Alonso. "La Escuela de Enfermería del Hospital Santo Tomás." Se encuentra en: http://www.alonsoroy.com/med/ med07.html. Consultado: 05/07/2005

- Sáenz, Rosa. Entrevista concedida a Ana Luisa Velandia, en Bogotá, en 1989.

- Velandia Mora, Ana Luisa (1995) Historia de la Enfermería en Colombia. Bogotá: Ediciones de la Universidad Nacional de Colombia. Etapa 1935 - 1954. Págs. 77 - 106.

- Velandia Mora, Ana Luisa (1995) Concha Romero: un modelo y un reflejo de su época. Avances en Enfermería. Volumen XIII, No. 1. Págs. 13-18.

- Velandia Mora, Ana Luisa. "Influencias étnicas en la enfermería latinoamericana". Publicado por la Revista Temperamentvm. 2005. ISSN: 1699 - 6011. http://www.index-f.com/temperamentum/1revista/1_sumario.php

- Velandia Mora, Ana Luisa. La Facultad de Enfermería de la Universidad Nacional de Colombia en el Siglo XX. Bogotá: Universidad Nacional de Colombia Unibiblos, 2006. 
- Velandia Mora, Ana Luisa. "Organizaciones interamericanas en la Enfermería en Colombia". Ponencia presentada en el XIII Congreso Nacional de Historia. Bucaramanga, Agosto 22 a 25 de 2006, b. Memorias en CD. ISBN 958 - 8187 - 55 - 9

- Velandia Mora, Ana Luisa (2009) "Helen Howitt: una semilla canadiense en la enfermería latinoamericana”. EN: Revista Avances en Enfermería. Vol. XXVII, No. 1, Enero - Junio.

- Velandia Silva, Geraldinne Marcela (2008) Repercusiones de la higiene pública y la Escuela Superior de Higiene en la formación del personal de enfermería en Colombia (1935 1960). Trabajo de Grado para optar al título de Enfermera.
Bogotá: Facultad de Enfermería, Universidad Nacional de Colombia.

- Wiesner, Lotti. Reseña Histórica de la Carrera de Enfermería en la Universidad Nacional de Colombia. Documento para la celebración de los 50 años de la Facultad. Bogotá, agosto de 1975. Págs. 51 - 55.

- Wiesner, Lotti (1976) "Desarrollo histórico de la carrera de enfermería de la Universidad Nacional de Colombia". Revista ANEC, Bogotá, Año VII, No. 17. Enero - abril. Págs. 51 - 55. - http://foundationnysnurses.org/collections/bellevuefa.htm Consultada: 31/01/2008.

${ }^{\prime}$ Los programas de las Escuelas Normales eran paralelos a los del bachillerato, también con seis años de duración; no abordaban la pedagogía sino hasta al tercer año. En 1951 se extendió a cuatro años los estudios para obtener el diploma de las Escuelas Normales Rurales que se habían iniciado en 1934 para darle formación al campesinado. En algunos casos esta formación fue aceptada para ingresar a estudiar enfermería.

${ }^{2}$ Notas de la traductora: Ana Luisa Velandia

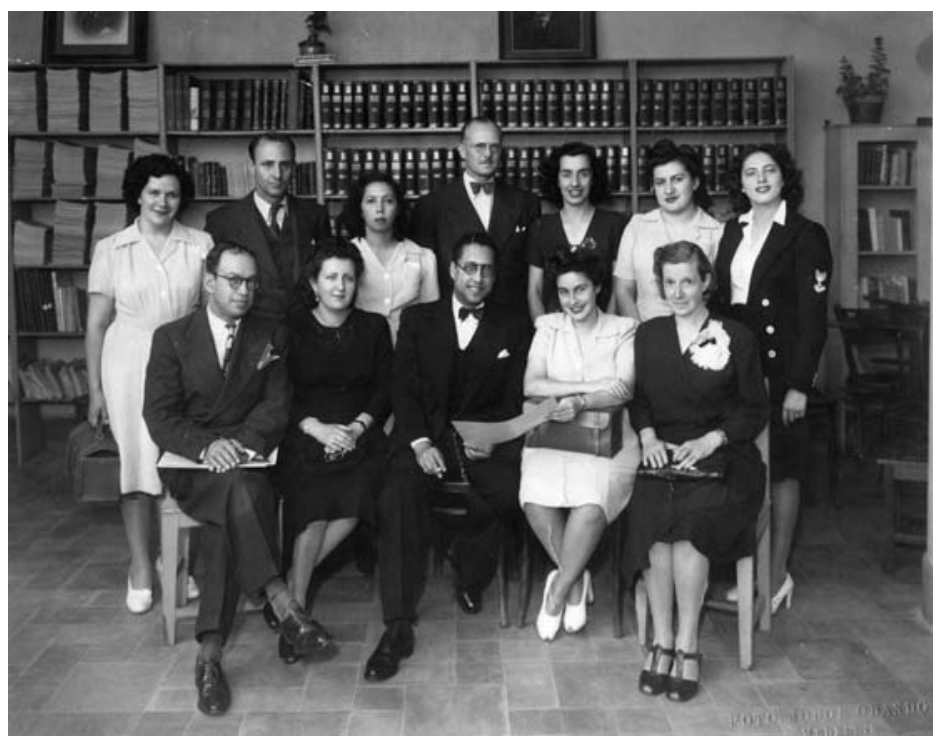

Funcionarios del Servicio Cooperativo Interamericano de Salud Pública. Sentada a la derecha, Helen Howitt. Foto Archivo de la Autora, donación de Lelly Álvarez de Espitia.

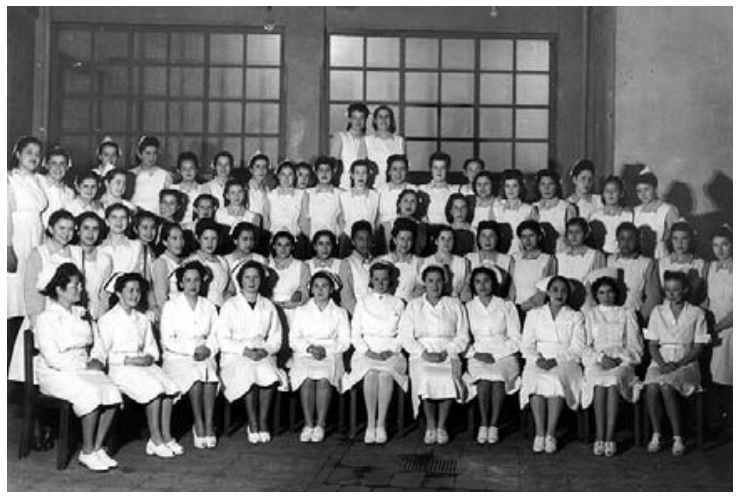

Celebración de Aniversario en la Escuela Nacional Superior de Enfermeras. Estudiantes, Profesoras y su Directora, Helen Howitt, sentada en el centro. Foto Archivo de la Autora, donación de Lelly Álvarez de Espitia. 